\title{
Digital Image Segmentation in Matlab: A Brief Study on Otsu's Image Thresholding
}

\author{
Md. Abu Bakr Siddique ${ }^{1 *}$, Rezoana Bente Arif ${ }^{1 @}$, Mohammad Mahmudur Rahman Khan ${ }^{2 \#}$ \\ ${ }^{1}$ Dept. of EEE, International University of Business Agriculture and Technology, Bangladesh \\ ${ }^{2}$ Dept. of ECE, Mississippi State University, Mississippi State, MS 39762, USA \\ absiddique@iubat.edu*, rezoana@iubat.edu ${ }^{@}$, mrk303@msstate.edu $^{\#}$ \\ Corresponding Author: absiddique@iubat.edu*
}

\begin{abstract}
Thresholding is considered as a statisticaldecision making theory which can lessen the average error incurred in allocating pixels to two or more groups. The traditional Bayes decision rule can be applied with the prior knowledge of the Probability Density Function (PDF) of each class. It is surmised that a threshold resulting in the best class separation is the optimal one. In this paper, Otsu's thresholding for image segmentation has been implemented. The well-known Otsu's method is to learn a threshold that can maximize the between-class variance or equivalently make light of the within-class variance of the entire image. At first, a color image of a tree is taken. After that, the image is transformed into a grayscale image. Then in the first part, twolevel thresholding is conducted, and later on, three-level thresholding is also applied. Again, two-level thresholding, as well as three level thresholding, are also applied to some other images. Finally, the comparison is made between two level thresholding and three level thresholding.
\end{abstract}

Keywords-Image segmentation, Otsu's thresholding, Two level thresholding, Three level thresholding, Error minimization, Bayes decision, Probability density function (PDF).

\section{INTRODUCTION}

Image segmentation is one of the fundamental techniques used to subdivide an image into its integrant portions to extract relevant information from the image [1]. In short, image segmentation changes the illustration of an image to its simplified form which is further momentous and natural to analyze $[2,3]$. There are several practical application of image segmentation such as trace tumors and additional pathologies [4, 5], machine vision, object detection [6], face detection, Medical imaging [7, 8], study and diagnosis of anatomical construction [9], fingerprint recognition and video surveillance etc. Several techniques have been undertaken over the past decades for image segmentation including thresholding [10], edge-based segmentation [11] and compression based methods [12], etc. Several algorithmic techniques such as Artificial Neural Network [13], Convolutional neural Network [14], and K-nearest Neighbors [15] can also be applied in image processing techniques such as segmentation, thresholding and filtering.

Among all the methods of image segmentation, thresholding is the simplest, yet most important and useful way of portioning an image into a front part and a background. Thresholding transforms a grayscale image into a binary image depending on threshold values [2]. The crucial part of the thresholding technique is to choose an optimum threshold value when three or more level of thresholding is incorporated. Nowadays, several thresholding methods are used including Otsu's method [10], clustering [16] and the utmost entropy method [17], etc.

Otsu's technique is an incredibly straightforward histogram-based [18] thresholding approach designed for automatic image thresholding [16]. The Otsu's algorithm concludes that the image is subdivided into two major categories: 1) The foreground and 2) The background. The algorithm strives to detect the best threshold value that bifurcate the histogram into two subsections so that combined spread is minimum or comparably "between class variance" is maximum [10]. The expansion of the actual Otsu's thresholding to multilevel thresholding is known as multi Otsu thresholding [19].

In this paper, Otsu's multilevel thresholding is implemented for digital image segmentation. At first, twolevel thresholding is executed, and then three level thresholding is also applied to the same image. After that, two level and three level thresholding are performed on some other pictures. Finally, the comparison is made between two levels and three levels thresholding regarding their performances in divulging the detailing of the images.

\section{LITERATURE REVIEW}

Though in real-world case, image segmentation performed effective roles in several demanding applications such as image retrieval, automatic traffic control, medical imaging, object detection, and video surveillance etc, image segmentation is very challenging due to the corruption by artifacts such as partial volume effect, image noises and bias field effects, image degradation such as blurring, contrast or color imperfection, insufficient resolution, RF inhomogeneity, image texture or structure variability and complexity etc. There are several image segmentation methods such as clustering, thresholding, region based, watershed, edge-based, Partial Differential Equation (PDE) based, and Artificial Neural Network (ANN) based. Among these segmentation methods thresholding method is the straightforward and advantageous method as it is quite effective in overcoming the current segmentation challenges mentioned above.

Region-based image segmentation can correct intensity inhomogeneities of magnetic resonance images with 
promising performances [20]. Yuhui et al. introduced hierarchical and generalized FCM in image segmentation to correct distance sensitivity to outliers [21]. Huang et al. discussed multilevel thresholding based on Otsu's method with the quantum constituent part cloud optimization algorithm [22]. Image segmentation based on the deep convolutional neural network is introduced for better performances known as SegNet [23]. Again, the probabilistic aggregation model is capable of utilized to achieve better image segmentation performances [24]. Recently, image segmentation has achieved promising performances with deep learning techniques such as deep convolutional networks with fully connected CRFs [25], VNet [26] and U-Net [27]. Again, multi-level 3D Otsu thresholding method has achieved promising results in brain image segmentation [28].

The ANN-based method is very time-consuming as it wastes more time in training and it is a supervised method. If images have too many edges, the edge-based method leads to ineffective results. The Region-based method is very expensive as it wastes more time and memory. As PDE based method uses complex programs, its computational complexity is very high. Again, in the clustering method, we have to determine membership function and it's not a trouble-free undertaking to perform. Watershed method requires a complex calculation of gradients. By considering all these difficulties in all other segmentation methods, thresholding method is the user-friendly and highly advantageous method as it is an unsupervised method which does require any prior information of the datasets and takes less time and memory to work with.

\section{OTSU'S THRESHOLDING BASICS}

In digital image segmentation, Otsu's thresholding is extensively used for the curtailment of the gray level image into a meaningful binary image. Like any other unsupervised methods such as Fuzzy C- Means [29] and ADBSCAN [30] clustering, Otsu's thresholding is also unsupervised. Otsu's thresholding presumes that the image accommodates two categories of pixels: a) The foreground pixels and b) The background pixels. Otsu's thresholding tries to calculate the best threshold values to split up two portions in such a way so that their inter-class variance becomes maximum and hence, their combined spread is minimum.

Now, for a given threshold $\mathrm{k}$ (a specific gray level), two classes prior probabilities are [1, 31]:

$$
\begin{aligned}
& P_{1}(k)=\sum_{i=0}^{k-1} p_{i} \ldots \ldots \\
& P_{2}(k)=\sum_{i=k}^{L-1} p_{i} \ldots \ldots
\end{aligned}
$$

So the total likelihood is:
$P_{1}(k)+P_{2}(k)=1$

The class probability is assessed from L bins of the histogram by the iterative method.

While the class means are:

$$
\begin{aligned}
& m_{1}(k)=\sum_{i=0}^{k-1} i p_{i} / P_{1}(k) \ldots \ldots \\
& m_{2}(k)=\sum_{i=k}^{L-1} i p_{i} / P_{2}(k) \ldots \ldots \\
& m_{T}(k)=\sum_{i=0}^{L-1} i p_{i} \ldots \ldots(6)
\end{aligned}
$$

So the following relation can be easily assumed to express total mean:

$$
m_{T}(k)=P_{1}(k) \cdot m_{1}(k)+P_{2}(k) \cdot m_{1}(k)
$$

Like class probability, the class means be able to as well computed iteratively. This thought leads to an efficient algorithm.

Now, the two classes' variances can be expressed by [1]:

$$
\begin{aligned}
& \sigma_{1}^{2}(k)=\sum_{i=0}^{k-1}\left(i-m_{1}(k)\right)^{2} p_{i} / P_{1}(k) . \\
& \sigma_{2}^{2}(k)=\sum_{i=k}^{L-1}\left(i-m_{2}(k)\right)^{2} p_{i} / P_{2}(k)
\end{aligned}
$$

So the total variance is:

$\sigma_{T}{ }^{2}(k)=\sum_{i=0}^{L-1}\left(i-m_{T}(k)\right)^{2} p_{i}$

Otsu's thresholding approach comprehensively searches for the threshold value that lessens the intraclass variance, established as a weighted sum of the incongruity of the two portions:

$\sigma_{w}^{2}(k)=P_{1}(k) \cdot \sigma_{1}^{2}(k)+P_{2}(k) \cdot \sigma_{2}^{2}(k)$

The between class variance is:

$\sigma_{B}^{2}(k)=P_{1}(k) \cdot\left(m_{1}(k)-m_{T}(k)\right)^{2}+P_{2}(k) .\left(m_{2}(k)-m_{T}(k)\right)^{2}$

As stated by Otsu's algorithm, lessening the intraclass variance is equal to maximizing the interclass variance [10].

So the total class variance is:

$\sigma_{T}^{2}(k)=\sigma_{w}{ }^{2}(k)+\sigma_{B}{ }^{2}(k)$

The class separability is:

$$
\eta=\frac{\sigma_{B}{ }^{2}(k)}{\sigma_{w}{ }^{2}(k)}
$$


At first, a color image was chosen. Then the color image was converted into a grayscale image. The image histogram was then calculated for $i=0,1, \ldots, L-1$. Then for each $k, k=1,2, \ldots, L-2$, the prior probabilities were computed. Also, the mean values were calculated.

Then the $\sigma_{B}{ }^{2}(k)$ was determined for each $k$. The best possible threshold is $k^{*}$ such that $\sigma_{B}{ }^{2}\left(k^{*}\right)$ is the maximum.

For selecting two thresholds:

$\sigma_{B}^{2}\left(k_{1}^{*}, k_{2}^{*}\right)=\max _{0<k_{1}<k_{2}<L-1}\left\{\sigma_{B}^{2}\left(k_{1}, k_{2}\right)\right\}$

\section{IMPLEMENTATION AND DISCUSSION OF RESULTS}

To perform Otsu's multi-level thresholding, the following image is selected first as shown in figure 1 below.

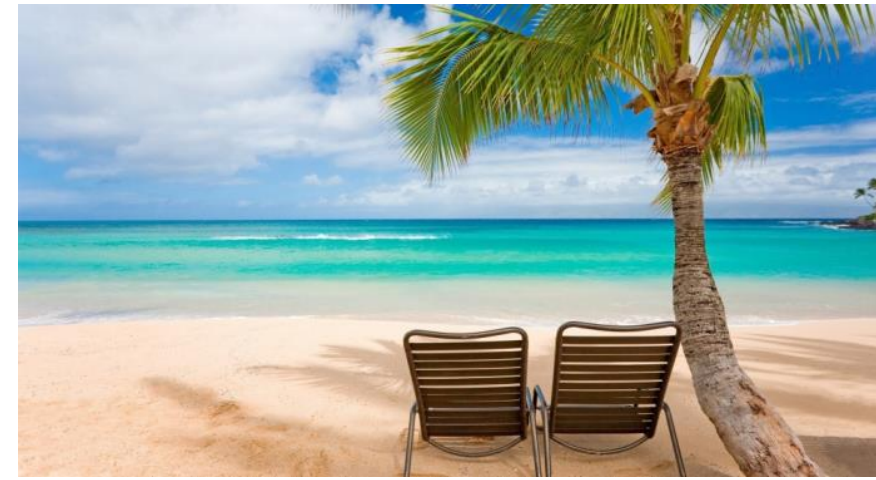

Fig. 1. Selected tree image to perform Otsu's thresholding

Now after converting the color image into the grayscale image, the two-level Otsu's thresholding is implemented. The result of this thresholding is exposed in figure 2. It is observed that the Otsu's thresholding has segmented the image into two levels as desired.

Now, the same image is chosen to perform three-level Otsu's thresholding. The output of this implementation is revealed in figure 3. Here, it is evident that the three-level thresholding is expressing more detail compared to the twolevel thresholding as three level thresholding has segmented the image into three levels.

Now, the two levels and three level thresholding are performed on several other images to see the difference between them as shown in Table 1 .

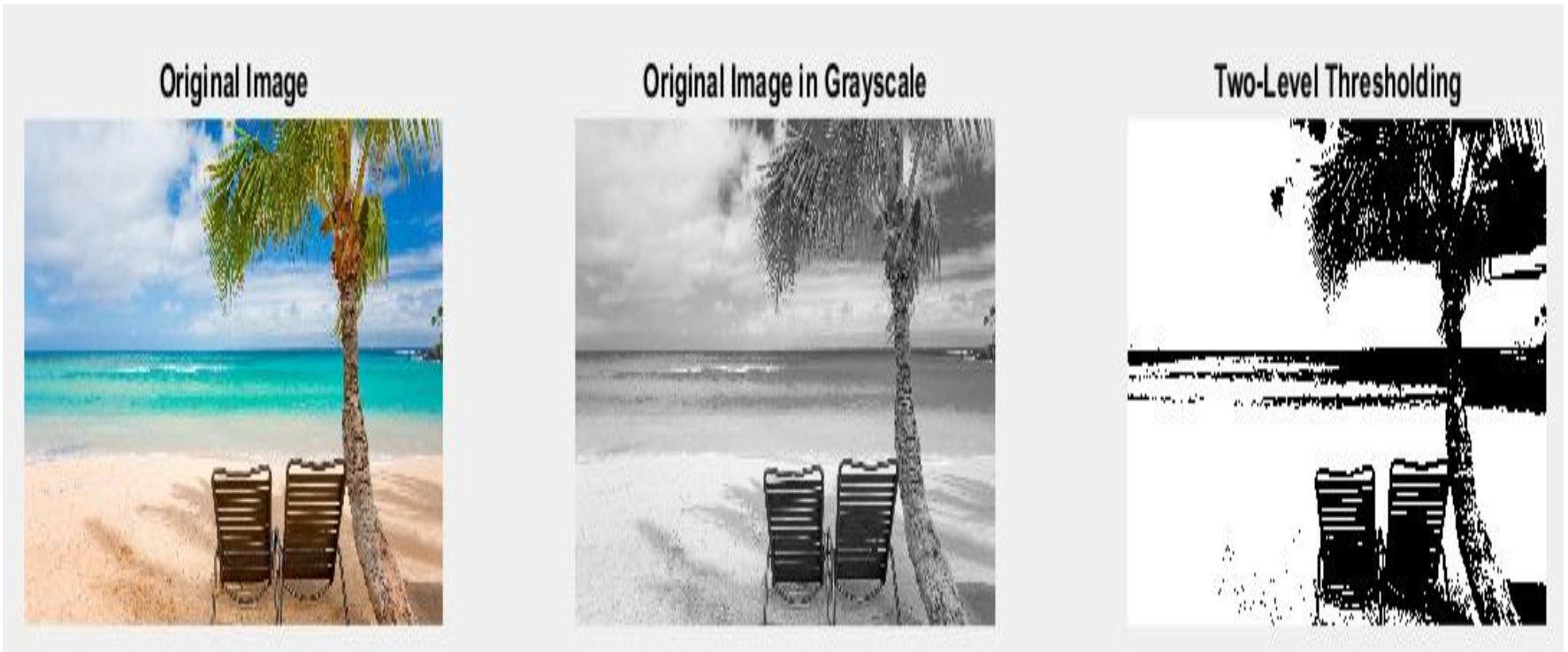

Fig. 2. The original tree image along with grayscale and two-level thresholding images 

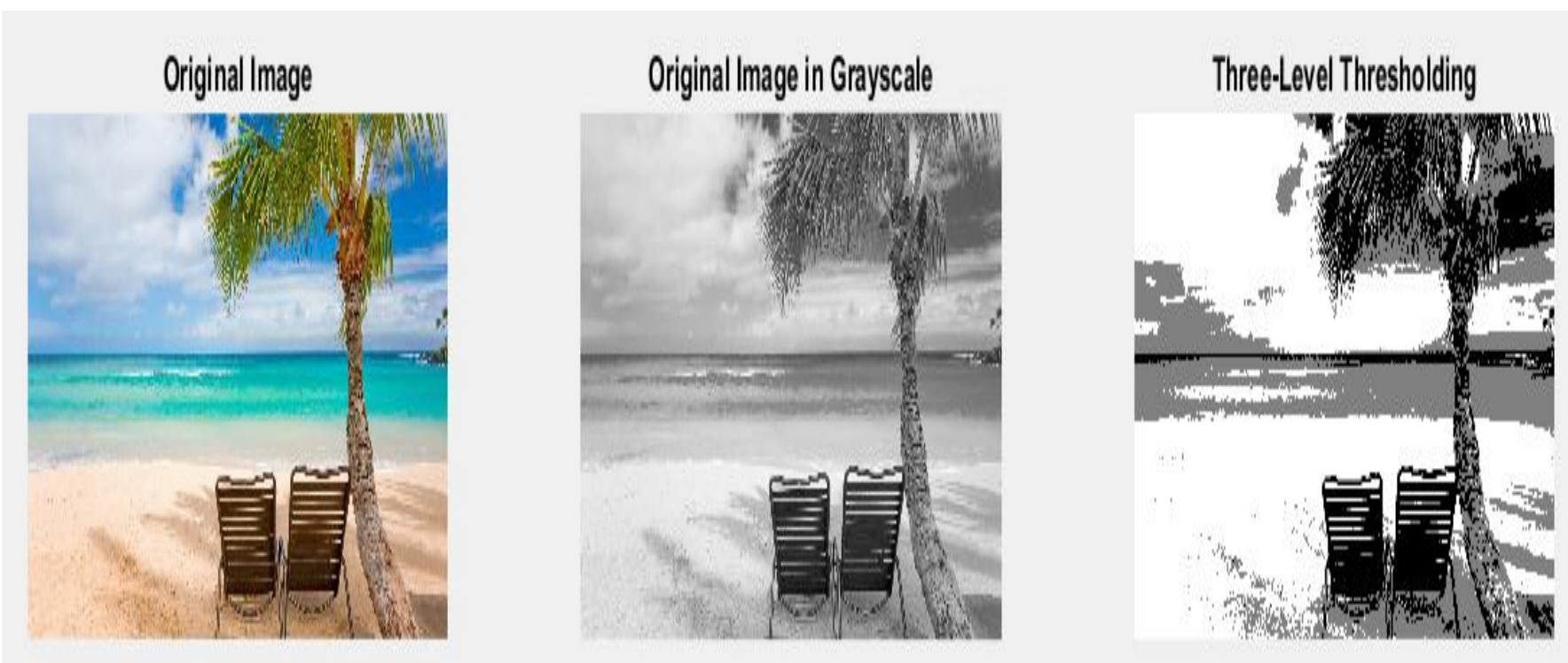

Fig. 3. The original tree image along with grayscale and three-level thresholding images

TABLE I. ORIGINAL IMAGE ALONG WITH GRAYSCALE, TWO LEVEL AND THREE LEVEL THRESHOLDING IMAGES

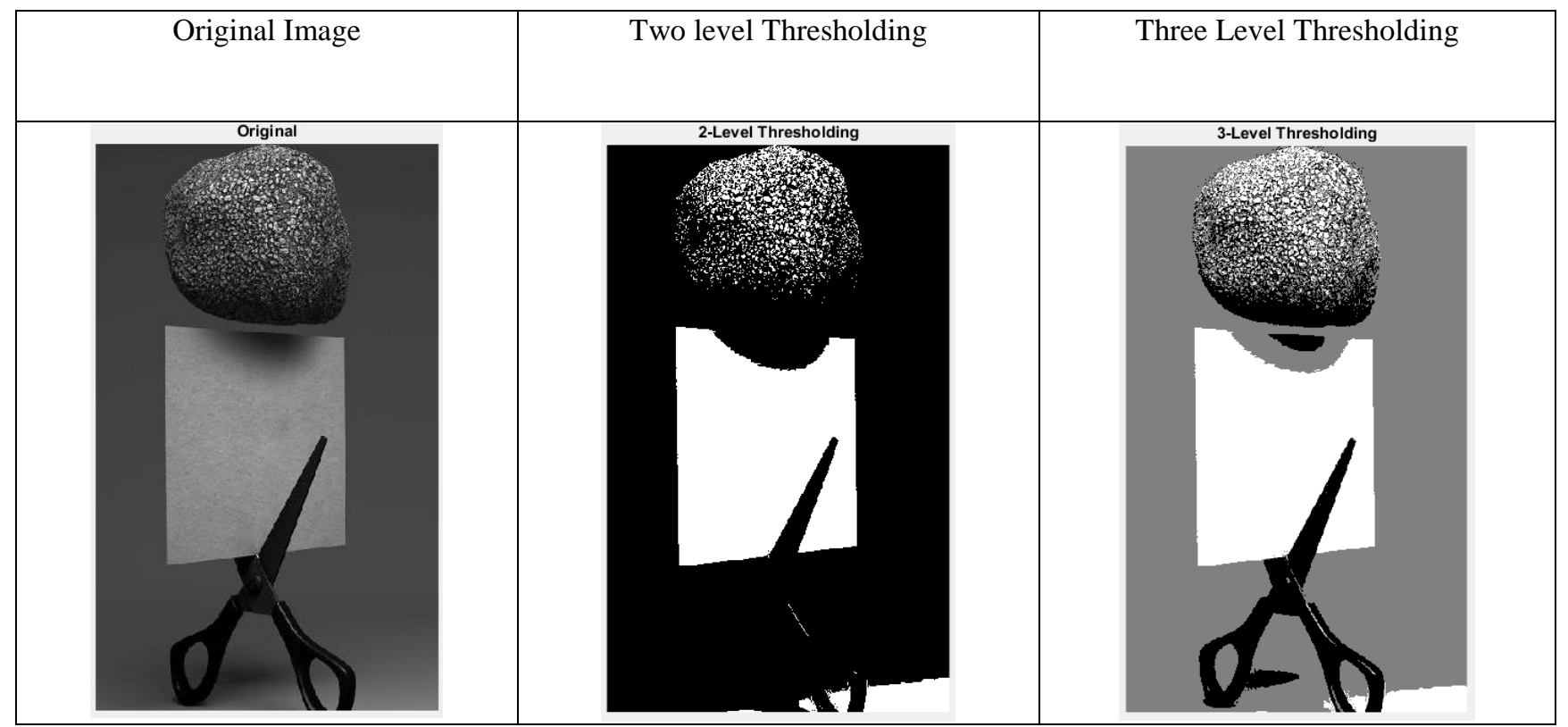




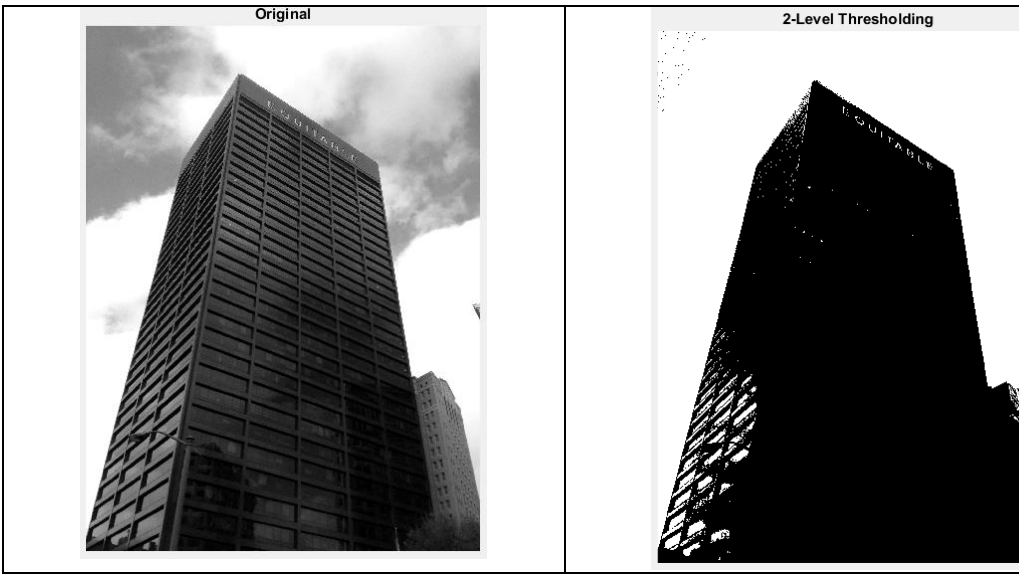

From the figures above it is observed that the rise of the threshold level of Otsu's thresholding algorithm increases the detailing of the image.

\section{CONCLUSION}

This paper presents Otsu's two level and three level thresholding' practical implementation of some images for image segmentation. It is observed that the three-level thresholding reveals more information about images compared to two-level thresholding. So it could be assumed that the detailing of images will be increased with the increment of the thresholding's level. In subsequent works of this series, some other improved thresholding techniques for image segmentation will be discussed.

\section{REFERENCES}

[1] R. C. Gonzalez and R. E. Woods, "Digital image processing," ed: Prentice hall New Jersey, 2002.

[2] L. G. Shapiro and G. C. Stockman, "Computer Vision, 2001, 279-325," ed: New Jersey, Prentice-Hall, ISBN 0-13-030796-3.

[3] L. Barghout and L. Lee, "Perceptual information processing system," ed: Google Patents, 2004.

[4] W. Wu, et al., "Brain tumor detection and segmentation in a CRF (conditional random fields) framework with pixel-pairwise affinity and superpixel-level features," International journal of computer assisted radiology and surgery, vol. 9, pp. 241-253, 2014

[5] E. B. George and M. Karnan, "MR brain image segmentation using bacteria foraging optimization algorithm," International journal of engineering and technology (IJET), vol. 4, pp. 295301,2012

[6] J. A. Delmerico, et al., "Building facade detection, segmentation, and parameter estimation for mobile robot localization and guidance," in Intelligent Robots and Systems (IROS), 2011 IEEE/RSJ International Conference on, 2011, pp. 1632-1639.

[7] D. L. Pham, et al., "Current methods in medical image segmentation," Annual review of biomedical engineering, vol. 2, pp. 315-337, 2000.

[8] M. Forouzanfar, et al., "Parameter optimization of improved fuzzy c-means clustering algorithm for brain MR image segmentation," Engineering Applications of Artificial Intelligence, vol. 23, pp. 160-168, 2010.

[9] S. Kamalakannan, et al., "Double-edge detection of radiographic lumbar vertebrae images using pressurized open DGVF snakes," IEEE Transactions on Biomedical Engineering, vol. 57, pp. 1325-1334, 2010.
[10]

N. Otsu, "A threshold selection method from gray-level histograms," IEEE transactions on systems, man, and cybernetics, vol. 9, pp. 62-66, 1979.

[11] O. Wirjadi, "Survey of 3d image segmentation methods," 2007.

[12] H. Mobahi, et al., "Segmentation of natural images by texture and boundary compression," International journal of computer vision, vol. 95, pp. 86-98, 2011.

[13] Md. Abu Bakr Siddique, et al., "Study and Observation of the Variations of Accuracies for Handwritten Digits Recognition with Various Hidden Layers and Epochs using Neural Network Algorithm," in 2018 4th International Conference on Electrical Engineering and Information \& Communication Technology (iCEEiCT), 2018, pp. 118-123.

[14] Rezoana Bente Arif, et al., "Study and Observation of the Variations of Accuracies for Handwritten Digits Recognition with Various Hidden Layers and Epochs using Convolutional Neural Network," in 2018 4th International Conference on Electrical Engineering and Information \& Communication Technology (iCEEiCT), 2018, pp. 112-117.

[15] Mohammad Mahmudur Rahman Khan, et al., "Study and Observation of the Variation of Accuracies of KNN, SVM, LMNN, ENN Algorithms on Eleven Different Datasets from UCI Machine Learning Repository," in 2018 4th International Conference on Electrical Engineering and Information \& Communication Technology (iCEEiCT), 2018, pp. 124-129.

[16] M. Sezgin and B. Sankur, "Survey over image thresholding techniques and quantitative performance evaluation," Journal of Electronic imaging, vol. 13, pp. 146-166, 2004.

[17] Y. Zhang and L. Wu, "Optimal multi-level thresholding based on maximum Tsallis entropy via an artificial bee colony approach," Entropy, vol. 13,pp. 841-859, 2011.

[18] A. dos Anjos and H. Shahbazkia, "Bi-Level Image Thresholding-A Fast Method," in BIOSIGNALS (2), 2008, pp. 70-76.

[19] P.-S. Liao, et al., "A fast algorithm for multilevel thresholding," J. Inf. Sci. Eng., vol. 17, pp. 713-727, 2001.

[20] C. Li, et al., "A level set method for image segmentation in the presence of intensity inhomogeneities with application to MRI," IEEE Transactions on Image Processing, vol. 20, p. 2007, 2011.

[21] Y. Zheng, et al., "Image segmentation by generalized hierarchical fuzzy C-means algorithm," Journal of Intelligent \& Fuzzy Systems, vol. 28, pp. 961-973, 2015.

[22] Y. Huang and S. Wang, "Multilevel thresholding methods for image segmentation with Otsu based on QPSO," in Image and Signal Processing, 2008. CISP'08. Congress on, 2008, pp. 701705 .

[23] V. Badrinarayanan, et al., "Segnet: A deep convolutional encoder-decoder architecture for image segmentation," arXiv preprint arXiv:1511.00561, 2015.

[24] S. Alpert, et al., "Image segmentation by probabilistic bottomup aggregation and cue integration," IEEE Transactions on Pattern Analysis and Machine Intelligence, vol. 34, pp. 315327,2012 
[25] L.-C. Chen, et al., "Deeplab: Semantic image segmentation with deep convolutional nets, atrous convolution, and fully connected crfs," IEEE Transactions on Pattern Analysis and Machine Intelligence, vol. 40, pp. 834-848, 2018.

[26] F. Milletari, et al., "V-net: Fully convolutional neural networks for volumetric medical image segmentation," in $3 D$ Vision (3DV), 2016 Fourth International Conference on, 2016, pp. 565-571.

[27] O. Ronneberger, et al., "U-net: Convolutional networks for biomedical image segmentation," in International Conference on Medical image computing and computer-assisted intervention, 2015, pp. 234-241.

[28] Y. Feng, et al., "A multi-scale 3D Otsu thresholding algorithm for medical image segmentation," Digital Signal Processing, vol. 60, pp. 186-199, 2017.

[29] M. Siddique, et al., "Implementation of Fuzzy C-Means and Possibilistic C-Means Clustering Algorithms, Cluster Tendency Analysis and Cluster Validation," arXiv preprint arXiv:1809.08417, 2018.

[30] Mohammad Mahmudur Rahman Khan, et al., "ADBSCAN: Adaptive Density-Based Spatial Clustering of Applications with Noise for Identifying Clusters with Varying Densities," in 2018 4th International Conference on Electrical Engineering and Information \& Communication Technology (iCEEiCT), 2018, pp. 107-111.

[31] R. C. Gonzalez, et al., Digital Image Publishing Using MATLAB: Prentice Hall, 2004. 